Marquette University

e-Publications@Marquette

College of Education Faculty Research and

Publications

Education, College of

8-1-2014

\title{
The Culturally-adapted Early Pathways Program for Young Latino Children in Poverty: A Randomized Controlled Trial
}

\author{
Michael P. Fung \\ Marquette University \\ Robert A. Fox \\ Marquette University, robert.fox@marquette.edu
}

Accepted version. Journal of Latina/o Psychology, Vol. 2, No. 3 (August 2014): 131-145. DOI. (C) 2014 American Psychological Association. Used with permission.

This article may not exactly replicate the final version published in the APA journal. It is not the copy of record. 


\begin{abstract}
This study used a randomized control design with treatment and wait-list conditions to evaluate the efficacy of a culturally-adapted version of the Early Pathways program (EP; Author citation, 2014), an in-home, parent-child therapy program with 137 at-risk Latino children under the age of six referred for severe behavior and emotional problems, such as aggression, oppositional behavior, self-injury and property destruction. EP directly engaged the parent-child dyad, emphasizing parent-directed training, child led play, psychoeducation, , and cognitivebehavioral strategies. Cultural modifications included establishing community partnerships to identify Latino family needs, translation of materials, offering bilingual services, acculturation assessment, and cultural competence training. Multivariate analyses of covariance (MANCOVA) revealed significant differences between the immediate and delayed treatment group on all post-test measures with the pre-test scores as covariates. After the delayed group completed treatment, repeated measures, multivariate analyses of variance (MANOVA) showed significant improvement for both groups on all measures with maintenance at four-to-six week follow-up. Outcomes included reduced child behavior problems, increased child pro-social behaviors, improved caregiver limit setting, enhanced caregiver nurturing, improved parent-child relationships, and a decrease in clinical diagnoses following treatment. This study highlights the efficacy of using culturally-adapted early intervention services for young Latino children in poverty referred for ignificant behavior and emotional problems.
\end{abstract}

Keywords: Behavior problems, young children, Latino, poverty 
There is a significant need for appropriate and effective mental health services within Latino communities, particularly for families with young children under the age of six. This need is highlighted by three widely acknowledged premises: (a) a rapid increase in the Latino population and an associated increase in poverty and mental health issues; (b) the unique qualities of Latino cultural values and their impact on effective mental health treatment; and (c) a limited number of Parent-Child Therapy (PCT) programs adapted for Latino families.

As the Latino population continues to grow, the poverty rate of Latino families has risen along with it. Between 2000 and 2010, the overall poverty rate for Latinos in the United States increased from $21.2 \%$ to $26.6 \%$, reflecting a total increase from $7,155,000$ to $13,386,000$ Latino individuals living in poverty (U.S. Census Bureau, 2001; U.S. Census Bureau, 2011). These statistics, however, do not fully capture the enormity of the poverty epidemic among Latino families with young children. In 2011, an estimated 1,147,503 Latino families with children under the age of six were living in poverty, representing $47 \%$ of Latino families living in poverty in the United States. Similarly, $37 \%$ of all Latino children under the age of six in the United States - an estimated 2,243,742 children - were living in poverty (U.S. Census Bureau, 2011).

This increase in poverty has engendered significant barriers to accessing mental health treatment (Alegría et al., 2008). For example, Latinos have the highest uninsured rate $(30.7 \%)$ in the United States compared to $11.7 \%$ of Caucasians, $20.8 \%$ of African-Americans, and $18.1 \%$ of Asian Americans (U.S. Census Bureau, 2011). Alegría et al., (2008) also found that $63.7 \%$ of Latinos suffering from depression did not access appropriate mental health care compared to $58.8 \%$ of African-Americans, $68.7 \%$ of Asian Americans, and $40.2 \%$ of Caucasians.

In order to provide effective services for this population, it is necessary to have a thorough understanding of Latino cultural values and their impact on parenting young children. 
Several constructs have been identified that describe Latino cultural values, including familismo, machismo, marianismo, respeto, personalismo, and simpatía (Barker, Cook, \& Borrego, Jr., 2010). Familismo is generally defined as the Latino emphasis on family unity and collectivism (Calderón-Tena, Knight, \& Carlo, 2011). Respeto encompasses a diverse array of values, including obedience to one's parents and upholding family honor through appropriate behavior, and it is also typically seen as the foundation for a hierarchical understanding of social relationships within Latino culture (Barker et al., 2010). Marianismo primarily refers to Latina gender roles related to the family, identifying women as the primary caregiver and nurturer within the home who will sacrifice for her family, which at times carries both positive connotation and challenges for Latina women (Castillo \& Cano, 2007). Similarly, machismo refers to both positive and negative characteristics of Latino men, including rigid gender roles and hypermasculinity, but also chivalry, courage, and being the head of the family (Arciniega, Anderson, Tovar-Blank, \& Tracey, 2008). Definitions of personalismo and simpatía tend to intersect, referring to the desire for warm, trusting relationships and a socially amicable persona, respectively (Barker et al., 2010).

Two meta-analytic reviews found that these cultural values play a significant role in therapy, and being mindful of these values supports effective cultural adaptation of therapeutic treatment programs and strategies (Griner \& Smith, 2006; Smith, Domenech-Rodríguez, \& Bernal, 2011). For example, familismo has been associated with higher levels of maternal nurturing behaviors and emotional support for adolescents (Calderón-Tena et al., 2011), as well as providing a protective influence on substance use among adolescents (Unger et al., 2002) and suicide attempts among Latina teens (Peña et al., 2011). In addition, Latino parents have identified respeto as an underlying theme of parent-child interactions, with a direct relationship 
to parental discipline (Arcia \& Johnson, 1998). Further, personalismo and simpatía have been found relevant to mental health outcomes based on several studies. For example, studies show that Latino families identify with their specific health care provider (i.e., physician) rather than a group practice, continue to see their provider despite relocation, and even stop pursuing health care if their provider is no longer available (Grossman, 1994). In fact, Foucault and Schneider (2009) conducted a study in the Dominican Republic and all but one of the mothers who were contacted in person participated in the study while $47 \%$ of mothers who were contacted by school letters participated in the program, highlighting the importance of direct personal contact with Latino participants.

Despite recognition of the influence of culture on therapy, there is a paucity of research examining the impact of cultural values on treatment for children under the age of six. In the past two decades, several PCT programs have been developed, such as Early Pathways (EP; Author citation, 2014); formally known as Parenting Young Children (PYC; Author citation, 2003), Parent Child Interaction Therapy (PCIT; Eyberg \& Boggs, 1989), Incredible Years Parenting Program (IYP; Webster-Stratton, 1992), and Triple-P Positive Parenting (Triple P; Sanders, 1999). Research on EP, PCIT, IYP, and Triple P has shown successful outcomes for children with behavior problems (Eyberg et al., 2001; Author citation, 2013; Roberts, Mazzucchelli, Studman, \& Sanders, 2006; Webster-Stratton, Reid, \& Hammond, 2004). While each of these programs has reported success with small portions of Latino populations, only a few have fully adapted a PCT program for Latino families and examined the effects of cultural modifications. Initial studies of culturally-adapted PCIT programs found support for such treatment among Puerto Rican and Mexican American families (Matos, Bauermeister, \& Bernal, 2009; McCabe \& Yeh, 2009). In addition, fairly successful outcomes have been found for less 
well-known program adaptations, such as Parenting Our Children to Excellence (PACE; Dumas, Arriaga, Moreland-Begle, \& Longoria, 2011) and Community Parent Education (COPE; Lakes et al., 2009). However, research with Latino children under the age of six is in its incipient stage, and none of these existing studies have evaluated in-home treatment for low-income Latino families to assess whether an outreach format offers the potential for even greater success.

This is particularly concerning considering the aforementioned high poverty rate among Latino families with young children, and that these at-risk children have a significantly greater risk for poorer social and emotional outcomes (Ackerman, Brown, \& Izard, 2003). Research has found that up to $36 \%$ of preschool children from families in poverty exhibit behavior problems (Qi \& Kaiser, 2003). These negative outcomes are due, in part, to a lack of availability and accessibility to mental health services (Spencer, Kohn, \& Woods, 2002). Research also has shown that families with low socioeconomic status (SES) often drop out of PCT treatment due to contextual factors, such as loss of phone services, child illnesses, and financial and family crises, as well as frequent relocation (Author citation, 1999).

In response to these challenges, there has been a gradual increased emphasis on providing treatment for young children in poverty. Fernandez, Butler, and Eyberg (2011) conducted a pilot study of PCIT with African American children from low-income families and found successful outcomes for children who completed treatment. Concurrently, there has been a recent trend to offer school-based services in an attempt to decrease the contextual barriers for at-risk children. For example, Webster-Stratton, Reid, and Stoolmiller (2008) provided group PCT services to parents of children from low-income families who were enrolled in Head Start, kindergarten, and first-grade programs; improved child behavior was reported. Brotman et al. (2011) also examined a school-based, group PCT program for children in prekindergarten from low-income 
families and reported a significant decrease in behavior problems. In addition, Breitenstein et al. (2007) reported positive outcomes for a group PCT program that was adapted for at-risk African American Head Start students while Gross et al. (2009) reported similar improvements in child behavior following a group PCT program for at-risk African American and Latino children in day care centers. These efforts reflect the growing realization that at-risk, young children require services tailored to their specific needs, which requires innovative intervention methods.

Despite their success, each of these programs offered treatment in community organizations or schools, which may not be the ideal settings for low-income families considering the contextual factors (e.g., transportation, maintaining clinic appointments) that may prevent families from regularly attending treatment and may contribute to a high attrition rate. As an alternative treatment setting, Wood, Barton, and Schroeder (1988) pointed out that in-home therapy has several major advantages including the ability to better tailor services to the unique needs of each family, an opportunity to obtain rich information on family dynamics and behaviors as they naturally occur, and the ability to provide services to individuals who would otherwise be unable to attend sessions at a clinic or school. Author citation (2009) also noted that in-home therapy was particularly efficacious for children with behavioral concerns as the behaviors could be addressed and corrected as they naturally occurred in session. In fact, inhome therapy was just as effective as residential care for behaviorally troubled children and is a recommended modality due to reductions in restrictiveness and cost (Barth et al., 2007).

The EP program uniquely offers in-home parent-child therapy for young children, focusing on parent-directed training and child behavior activities to decrease child behavior problems. Multiple studies have reported the effectiveness of the EP model for young children with behavior problems, notably including at-risk children living in poverty (Author citation, 
2009; Author citation, 2013), children with developmental delays (Author citation, 2009) and children from different ethnic backgrounds (Author citation, 2014).

\section{Purpose of the Study}

The purpose of this study was to culturally adapt, implement, and evaluate a PCT program for low-income Latino families with children under the age of six who were referred for severe behavior problems. The EP program served as the original PCT format to be adapted because it offered unique, in-home treatment for low-income families who may not otherwise be able to attend therapy. This factor made EP an ideal program format for Latino families considering the aforementioned poverty statistics and related barriers to treatment. In addition, while previous EP research has reported positive outcomes, the present study attempted to determine whether EP was the primary cause of change in child behavior problems by using a waitlist-control group within a randomized controlled design.

\section{Research Questions}

1. Did Latino children referred for mental health services improve from pre-test to post-test on all measures following their participation in a culturally-adapted PCT program compared to a delayed control group?

2. Did the delayed control group subsequently improve after participation in the program and were these changes maintained at four-six week follow-up in both groups?

\section{Method}

\section{Participants}

Participants were 137 Latino children under the age of six from low-income families who were referred for severe behavior problems. Services were provided in participant homes, which were located within a large urban community in the upper Midwest. Eligibility criteria included: 
(a) the child was under 72 months of age; (b) the child was referred for a significant mental health concern such as aggression, destructiveness, hyperactivity, self-injury, or separation anxiety; (c) the child did not have a serious physical disability, significant health concern, or meet the criteria for moderate to profound intellectual disability or autistic spectrum disorder; however the child may have had a developmental delay (e.g., cognitive, speech, motor); (d) at least one of the child's parents was Latino; (e) the family met the federal criteria for living in poverty (e.g., eligible for public assistance programs); (f) the primary caregiver signed an IRBapproved informed consent form for the child and family to participate in this study. If the parent or caregiver declined to participate, they still received the full EP treatment program but their information was omitted from this study.

Of the 137 participants, there were 100 boys (73\%) and 37 girls (27\%). Child ethnicities included Mexican (48.5\%), Puerto Rican (27.2\%), other (14.7\%; e.g., Dominican, Central American, Spanish), and mixed ethnicities (9.6\%; e.g., Mexican/Puerto Rican, Mexican Dominican). The majority of caregivers were mothers $(94.9 \%)$, and $60.6 \%$ of primary caregivers were unemployed. Fifty-six percent of primary caregivers were single, $35 \%$ were married, and $9 \%$ were separated/divorced. Of the participants, $47.4 \%$ completed the treatment program in Spanish, $44.5 \%$ in English, and $8 \%$ in Spanish and English. Average primary caregiver age was 28.87 years $(S D=6.88)$ and average child age was $3.89(S D=1.11)$. A total of $46.7 \%$ of the children were diagnosed with a developmental delay, of which $82 \%$ were language disorders. At intake, $88.7 \%$ of children received an initial psychiatric diagnosis. Disruptive Behavior Disorder NOS was the most common primary diagnosis at intake (45.1\%); additional primary diagnoses included Oppositional Defiant Disorder (23.8\%), Adjustment Disorder (9.8\%), other (8.1\%; e.g., Separation Anxiety, Reactive Attachment Disorder), Parent-Child Relationship Problem (6.6\%), 
Early Pathways for Young Latino Children in Poverty 9

Post-Traumatic Stress Disorder (4.1\%), and Attention Deficit Hyperactivity Disorder (2.5\%).

These psychiatric diagnoses, while required for insurance reimbursement, were carefully reviewed by a licensed psychologist to ensure accuracy and prevent over pathologizing of these very young children.

\section{Procedure}

This project was approved by the Internal Review Board of a private university. A convenience sample of consecutive Latino children referred from over 50 agencies and individual providers to EP was used. A random-number generator assigned 1000 numbers (0 or 1) to a Microsoft Access case management database. Children were entered into the database in the order that they were referred and determined to be eligible to participate in the study, thus ensuring random assignment to either the immediate $(\mathrm{n}=80)$ or delayed treatment groups $(\mathrm{n}=$ 57). However, if children were identified at intake as having a significant trauma history or a serious behavior problem that compromised the safety of the child or others, they received immediate treatment regardless of randomization protocol to ensure participant safety.

Project data were collected over a one and a half year period. All participants signed an informed consent form describing the purpose, risks, and benefits of treatment prior to completing the intake evaluation. The immediate treatment group completed an intake, treatment, post-test, and four-six week follow-up sessions. The delayed treatment group completed an intake, waited four to six weeks, and repeated the pre-test measures during a second intake before following the same treatment and assessment protocol as the immediate treatment group. Booster sessions were provided at the request of families after the follow-up session. A total of 26 booster sessions with eight families were completed.

\section{Instruments}


Intake Form (IF). The IF was used to collect demographic information about the referred child (e.g., gender, date of birth, siblings), and the family). The IF also was used to collect information about the child's birth and health history, and involvement with child protective services and other service providers. The IF helped determine the frequency and nature of the child's referral concerns, possible contributing factors, and how the caregivers were presently responding to the referral concerns.

Short Acculturation Scale for Hispanics (SASH). The SASH (Marín, Sabogal, Marín, Otero-Sabogal, \& Perez-Stable, 1987) is a 12-item acculturation scale that is designed to measure the acculturation status in Latino populations. The scale contained 12 items across three factors: Language Use (e.g., "In general, what language do you read and speak?"), Media (e.g., “In what languages are the T.V. programs you watch?”), and Ethnic and Social Relations (e.g., "Your close friends are..."). Scores ranged from 1 to 5 for items one through eight $(1=$ Only Spanish, 3 = Both Spanish and English equally, $5=$ Only English $)$ and for items 9 to $12(1=$ All Latinos/Hispanics, 3 = About half and half, $5=$ All Americans). Norms were developed using 363 Hispanic and 228 non-Hispanic males and females. The Hispanic group ranged from 15-75 years of age $(S D=11.6)$. The coefficient alpha for the overall scale is .92. Coefficient alphas for the three subscales are .90 ("Language"), .86 ("Media"), and .78 ("Ethnic Social Relations").

Early Childhood Behavior Screen (ECBS). The ECBS (Author citation, 2012) is a 20item self-report screening instrument developed specifically for very young children (0 to fiveyears-old) from low-SES backgrounds. The ECBS includes 10 positive behavior items (e.g., "listens to you") and 10 challenging behavior items (e.g., "hits others") and is written at a 3.9 grade level. Caregivers rated each item based on their perception of their child's behavior over the past week using a three-point Likert scale $(1=$ almost never, $2=$ sometimes, $3=$ often $)$. 
Total scores on both the Positive Behavior Scale (PBS) and Challenging Behavior Scale (CBS) ranged from 10 to 30 with higher scores indicating a greater frequency of pro-social and challenging behaviors, respectively. Field-testing of the ECBS was conducted with a representative, diverse sample of 439 parents (16\% Latino) from a low-SES urban community. Internal consistencies using coefficient alphas were reported for the CBS (.87) and PBS (.92). The CBS demonstrated adequate levels of concurrent validity $(r=.75)$ with the Eyberg Child Behavior Inventory (ECBI; Eyberg \& Pincus, 1999) and adequate levels of sensitivity (82\%) and specificity $(80 \%)$ based on its relationship with the ECBI cutoff scores for clinical significance.

Parent Behavior Checklist (PBC). The PBC (Author citation, 1994) is a 32-item rating scale that is designed to measure the behaviors and expectations of parents of young children between the ages of one and five. For this project, two subscales were used from the PBC. The discipline subscale consisted of 10 items that assessed parental responses to the child's problem behaviors (e.g., "I yell at my child for whining"). The nurturing subscale consisted of 10 items that measured parent behaviors that promoted the child's psychological growth (e.g., "My child and I play together on the floor"). Items were rated using a four-point frequency scale (1 = almost never/never, 2 = sometimes, 3 = frequently, and $4=$ almost always/always). Total scores for discipline and nurturing ranged from 10 - 40, with higher scores indicating more frequent use of verbal and corporal punishment (e.g., yelling) and positive nurturing activities (e.g., reading with child), respectfully. From a representative sample of 1,140 mothers (<5\% Hispanic), internal consistencies using coefficient alphas were reported: Discipline $=.91$ and Nurturing $=$ .82. Test-retest reliabilities for the two subscales were: Discipline $=.87$ and Nurturing $=.81$.

Parent-Child Play Assessment (PCPA). Parents were instructed to play with their child with toys in the home while the clinician rated the quality of the parent-child interaction. Based 
on the work of Crawley and Spiker (1983), five dimensions of the child's behavior and six dimensions of the parent's behavior were rated using a three-point frequency scale $(1=$ poor, $2=$ fair, $3=$ good). For 142 independent observations (72 African American, 44 Latino, 19 Caucasian, 7 Mixed) by two clinicians from a previous study (Author citation, in press), kkappa coefficients were: child behaviors - positive affect $=.78$, negative affect $=.80$, interest in play $=$ .80 , initiates interactions $=.82$, social responsiveness $=.83 ;$ parent behaviors - parent leads $=$ .84 , parent engagement $=.73$, sensitivity to child $=.85$, expectations of child $=.85$, sets appropriate limits $=.82$, and reciprocity $=.83$. These coefficients indicated good agreement between observers (Viera \& Garrett, 2005). Separate total scores were computed for the five dimensions of the children's behaviors and the six dimensions of the parents' behaviors. Coefficient alphas for the present sample were .82 for the child behavior scale and .79 for the parent behavior scale.

Diagnostic and Statistical Manual of Mental Disorders (DSM; APA, 2000). In addition to the other four axes, the Global Assessment of Functioning (GAF) was completed. When two clinicians were present, each completed an individual GAF score. Based on 100 cases, the kappa coefficient for inter-rater reliability was .56, which indicates moderate agreement between observers (Viera \& Garrett, 2005).

Parent-Child Relationship Scale (PCRS). This scale provided a subjective and quantitative global assessment of the parent-child relationship on a scale of 0-100 with five behavioral anchors (poor, below average, average, good, and exceptional) at 20-point intervals (Author citation, 2003). For example, scores suggestive of a good relationship (e.g., thoughtful interactions, parent responsiveness, appropriate limit setting) ranged from 60-80. When two 
clinicians were present, each completed an individual PCRS score. For 101 cases, the kappa coefficient was .57, indicating moderate agreement between observers (Viera \& Garrett, 2005).

Family Satisfaction Survey (FSS). At the completion of the treatment program, a 7item anonymous survey was used to assess caregiver satisfaction with treatment. On a 7-point Likert rating scale, caregivers were asked to rate: quality of services received, how services contributed to their child's improvement, how the clinic helped them to improve management of their child, if caregivers would use the clinic again, current status of the child's referral concern, if caregivers would recommend the clinic to others, and the caregiver's confidence in managing their child's behavior. Internal consistency was $r=.82$ for the present sample.

\section{Early Pathways Program}

The EP program (Author citation, 2014) was adapted for home-based use with families in poverty from the evidence-based Parenting Young Children program (Author citation, 2003). The in-home intervention addressed several barriers to treatment attributed to poverty, including loss of phone services, financial and family crises, and frequent relocation (Author citation, 1999). Intake duration was approximately 90-120 minutes and treatment sessions typically lasted 60-90 minutes. To enhance rapport, clinicians who completed the intake session, also conducted the intervention and post-test session. Moreover, to avoid over-pathologizing during the intake, clinicians emphasized that child behavior problems are quite common in young children under the age of six, and diagnoses simply reflected an elevated level of frequency and severity of typical child behaviors. During early stages of treatment, caregivers were taught child-led play, a non-directive interaction where the child chooses and leads play while the caregiver follows and offers positive comments. The goal was to strengthen the parent-child relationship, and caregivers were encouraged to participate in this play at least 15 minutes each 
day. There were five additional components. First, psychoeducation regarding child development, and the development and maintenance of challenging behaviors was offered. Second, caregivers were taught the STAR technique (Author citation, 2003), a cognitivebehavioral strategy to manage parental thoughts and feelings and related responses to challenging behavior. Caregivers were instructed to stop (S) before reacting to challenging behaviors, think (T) about their thoughts and emotions, ask (A) themselves what a developmentally appropriate response would be, and finally respond $(\mathrm{R})$ to the child in a thoughtful manner. Third, appropriate expectations based on the child's developmental age were discussed with caregivers. Fourth, caregivers were taught to implement behavioral strategies, such as positive reinforcement and predictable home routines, to increase children's positive behaviors. Finally, strategies to manage challenging behaviors were introduced, including ignoring, redirection, and time-out.; all forms of verbal and corporal punishment were strongly discouraged. Clinicians provided caregivers with individualized, behavior treatment plans and a daily checklist to facilitate caregiver practice each week, which families completed and returned at the beginning of the subsequent session.

Cultural Adaptations. Cultural modifications followed the guidelines established by the ecological validity model (Bernal, Bonilla, \& Bellido, 1995) and the process model of Domenech-Rodríguez \& Wieling (2004). While their models deserve a more extensive description, in sum, the adaptation process involved addressing eight culturally-relevant content areas (e.g., language, concepts, context), as well as matching EP with the treatment population through partnerships with a community organizations to identify family needs and barriers along with concurrent implementation and adjustment of the intervention. Based on these guidelines and feedback from Latino families, the following protocol modifications were made: (a) 
established a community partnership with a large, non-profit organization in the area; (b) forward-backward translation of program materials to Spanish; (c) addition of bilingual clinicians and a Spanish interpreter to the clinical staff; (d) inclusion of an acculturation measure to identify adherence to cultural values; and (e) cultural competence training for clinicians.

The community partnership involved meetings with community pediatricians, nurses, and social workers at a large non-profit, health care organization to identify community needs, barriers, and program goals. Initial modifications were analyzed and adjusted throughout program duration. The Spanish interpreter was trained to maintain client confidentiality and interpreter familiarity with mental health terminology. Prior to conducting visits with nonSpanish speaking clinicians, the interpreter shadowed the primary bilingual clinician and discussed treatment protocol and terminology to ensure accuracy of translation. Cultural competence training for all staff and students included a review of Latino cultural constructs and their potential impact on Latino parenting. For example, clinicians were instructed that Latino caregivers may place a greater emphasis on familial rather than non-familial relationships, and treatment may involve extended family members (e.g., grandparents, aunts). In addition, clinicians were informed that they may need to spend additional time building rapport with the family at the outset of treatment due to the Latino preference for close, personal relationships with providers. Clinicians also were instructed that they may have to implement unique treatment strategies, such as physical (hugs) rather than verbal forms of praise, as well as focus on the potential impact of gender roles during treatment.

Clinician Training. One full-time bilingual licensed professional counselor and one bilingual doctoral student served as the primary clinicians on the project. In addition, three fulltime licensed professional counselors, three full-time counselors-in-training, two doctoral 
psychology students, and five master's level graduate students served as clinicians for the project. For non-Spanish speaking clinicians, the Spanish interpreter attended sessions to translate materials. A consulting psychologist and clinical director who were both familiar with urban Latino populations, though neither was bilingual, provided supervision. Senior clinicians and doctoral students trained novice clinicians on the treatment protocol using a three-step process: (a) novice clinicians received didactic training and read an extensive training manual on EP, (b) they shadowed senior clinicians on in-home visits, and (c) they gradually implemented in-home treatment protocol under supervision. Incoming clinicians also were trained on how to competently address barriers to treatment that arise while working with a diverse racial and ethnic population within an urban setting, with low-income backgrounds. For example, clinicians often conducted advocacy efforts (e.g., consulted with school administrators, met with social workers), adapted treatment protocol (e.g., no safe space for time-outs due to condition of the home), and adjusted appointment times because families in poverty received a myriad of services, often conflicting with treatment. Moreover, families in poverty often had multiple children and caregivers living in the home, requiring clinicians to engage everyone to establish consistency of the intervention strategies. When novice clinicians demonstrated appropriate professional demeanor, showed cultural sensitivity, and completed administrative documentation based on a treatment integrity checklist, they were allowed to facilitate individual cases. Each clinician received individual supervision and attended weekly case management meetings to discuss client progress, address concerns, and receive feedback on clinician performance.

\section{Results}

All results reflected intent-to-treat (ITT) analyses by including all families who had available data regardless of whether they dropped out of treatment. All families with complete 
data were considered to have completed treatment. This analysis is more conservative than a dose-effect comparison. A total of $26(32.5 \%)$ participants from the immediate group and 18 (31.6\%) participants from the delayed group dropped out prior to completing a post-test evaluation, and 18 participants from the immediate group and 16 participants from the delayed group did not complete a follow-up evaluation (see Figure 1). Treatment completers and dropouts were compared on demographics and pre-test measures. No differences were found on pre-test measures. On demographics, dropouts were more likely to be single $\left[\chi^{2}(2)=9.52, p<\right.$ $.01]$ and also more commonly spoke English $\left[\chi^{2}(1)=7.16, p<.01\right]$.

Immediate and delayed treatment groups were then compared on demographic, acculturation, and treatment adherence variables. The immediate treatment group had a larger number of employed parents $\left[\chi^{2}(1)=7.02, p<.01\right]$. Otherwise, there were no differences between groups on demographic or acculturation variables. Average program duration was 3.25 months $(S D=1.25)$ for the delayed treatment group and 2.7 months $(S D=1.58)$ for the immediate treatment group while treatment attendance was $81 \%$ for both groups. No significant differences between groups were found on treatment adherence variables.

Multivariate analyses of covariance (MANCOVA) were computed to determine if the immediate group differed from the delayed group on post-test measures with the pre-test scores as covariates. The MANCOVA tests showed a significant difference between the immediate and delayed groups on the ECBS measure with a large effect size $[F(2,81)=39.79, p<.001$, Cohen's $d=1.98]$. A review of univariate tests revealed significant differences on the ECBSPBS with a large effect size $[F(1,82)=19.02, p<.001$, Cohen's $d=.96]$ and the ECBS-CBS with a large effect size $[F(1,82)=72.32, p<.001$, Cohen's $d=1.88]$. Results also revealed a significant difference between the immediate and delayed groups on the overall PCPA measure 
with a large effect size $[F(2,73)=24.96, p<.001$, Cohen's $d=1.65]$. A review of univariate tests revealed significant differences on the PCPA child scale with a large effect size $[F(1,74)=$ 20.96, $p<.001$, Cohen's $d=1.07]$ and the PCPA parent scale with a large effect size $[F(1,74)=$ $50.14, p<.001$, Cohen's $d=1.65]$. For the combined PBC discipline and nurturing scales, a significant difference between the immediate and delayed groups was found with a large effect size $[F(2,81)=8.75, p<.001$, Cohen's $d=.93]$. A review of univariate tests revealed significant differences on the PBC discipline scale with a large effect size $[F(1,82)=14.61, p<.001$, Cohen's $d=.84]$ and the PBC nurturing scale with a medium effect size $[F(1,82)=7.38, p<.01$, Cohen's $d=.60]$. MANCOVA tests also revealed a significant difference between the immediate and delayed groups on the overall GAF and PCRS measures with a large effect size $[F(2,77)=97.16, p<.001$, Cohen's $d=3.18]$. A review of univariate tests revealed significant differences on the individual GAF measure with a large effect size $[F(1,78)=143.09, p<.001$, Cohen's $d=2.71]$ and the individual PCRS measure with a large effect size $[F(1,78)=132.92, p$ $<.001$, Cohen's $d=2.61]$. After the delayed treatment group completed EP, participants showed significant improvement similar to the immediate treatment group on all variables (see Table 1). Subsequently, repeated measures, multivariate analyses of variance (MANOVA) were conducted to determine if significant change was made from pre-test to follow-up for the overall sample of both groups. Results showed a significant time effect on the overall ECBS measure with a large effect size $[F(4,54)=38.22, p<.001$, Cohen's $d=3.37]$. A review of univariate tests revealed significant time effects on the ECBS-PBS with a large effect size $[F(2,114)=$ $33.96, p<.001$, Cohen's $d=1.54]$ and the ECBS-CBS with a large effect size $[F(2,114)=$ 97.95, $p<.001$, Cohen's $d=2.62$ ]. Results for the PCPA measure also showed a significant overall time effect with a large effect size $[F(4,39)=13.75, p<.001$, Cohen's $d=2.37]$. A 
review of univariate tests revealed significant time effects on the PCPA child scale with a large effect size $[F(2,84)=15.58, p<.001$, Cohen's $d=1.22]$ and the PCPA parent scale with a large effect size $[F(2,84)=28.16, p<.001$, Cohen's $d=1.64]$. A significant overall time effect with a large effect size was found on the combined PBC discipline and nurturing scales $[F(4,54)=$ $13.45, p<.001$, Cohen's $d=2.00$ ]. A review of univariate tests revealed significant time effects on the PBC discipline scale with a large effect size $[F(2,114)=28.25, p<.001$, Cohen's $d=$ 1.41] and the PBC nurturing scale with a medium effect size $[F(2,114)=4.26, p<.05$, Cohen's $d=.55]$. There also was a significant time effect on the overall GAF and PCRS measures with a large effect size $[F(4,53)=54.47, p<.001$, Cohen's $d=4.05]$. A review of univariate tests revealed significant time effects on the GAF measure with a large effect size $[F(2,112)=152.36$, $p<.001$, Cohen's $d=3.30]$ and the PCRS measure with a large effect size $[F(2,112)=132.45, p$ $<.001$, Cohen's $d=3.08]$. No differences between groups were found on any of the measures $(p$ $>.05)$. Pairwise comparisons of all measures revealed significant change from pre-test to posttest and maintenance of change from post-test to follow-up (see Table 2).

Of the $85.7 \%$ of children in the immediate treatment group who received a primary diagnosis at intake, $58.9 \%$ no longer met the criteria for a psychiatric diagnosis at post-test. Meanwhile, of the $92.9 \%$ of children in the delayed treatment group who received a primary diagnosis at intake, $90.9 \%$ still met the criteria for a psychiatric diagnosis at the second pre-test. A chi-square test revealed no differences between groups at pre-test $(p>.05)$. However, after the immediate group received treatment, the percentage of children with diagnoses was significantly greater in the delayed group $\left[\chi^{2}(8)=34.23, p<.001\right]$. After they both had received treatment, $67 \%$ of the delayed group no longer met criteria for a psychiatric diagnosis at post-test and a chi-square test between groups was not significant $(p>.05)$. 
Early Pathways for Young Latino Children in Poverty 20

Scores from each of the seven items (range 1 to 7 ) on the FSS were summed to create an aggregate score ranging from 7 (low satisfaction) to 49 (high satisfaction). The mean score at post-test was $46.67(S D=2.48)$ for the immediate group and $46.41(\mathrm{SD}=2.67)$ for the delayed group, indicating a high level of satisfaction in both groups following treatment.

\section{Discussion}

The present study developed, implemented, and analyzed a culturally-adapted version of the EP program for Latino families in poverty with children under the age of six who had been referred for severe behavior and emotional problems. Results revealed significant improvement on all measures along with and a high level of caregiver satisfaction with the intervention. These results support previous studies (Author citation, 2009; Author citation, 2013; Author citation, 2009) highlighting the clinical impact of EP as an early intervention to prevent the development of ingrained behavior patterns during later childhood and adolescence.

The use of a randomized, waitlist-control design highlights the contributory effect of the EP program to the successful outcomes of Latino children participants, further strengthening the research on EP with children from different ethnic backgrounds as initially identified by Gresl et al. (2014). Moreover, after the delayed group completed treatment, participants from both the immediate and delayed groups exhibited similar positive outcomes with successful maintenance of gains, importantly highlighting the long-term efficacy of the EP program. This long-term, sustainable impact is likely a result of the program's requirement that caregivers directly implement treatment strategies and participate fully in the intervention. By the end of treatment, caregivers had developed the skills to manage their children's behaviors independently. Also, younger children's behavior problems are more quickly addressed by consistent implementation of effective strategies than older children, whose behavior often has become more ingrained. 
The direct observation of child behavior in the family's natural environment provides compelling support for the in-home treatment format because clinicians were able to directly witness challenging behavior patterns and difficult parent-child interactions. Clinicians then were able to adapt treatment to each family's specific concerns rather than offer generic treatment strategies. The in-home structure also empowered caregivers to address their children's challenging behaviors in the most pragmatic setting possible while also overcoming the aforementioned barriers to treatment for families in poverty.

This study also adds to the growing body of literature (Dumas et al., 2011; Lakes et al., 2009; Matos et al., 2009; McCabe \& Yeh, 2009) suggesting that cultural modifications to evidence-based treatment are beneficial when offering early intervention services for culturally diverse participants. In addition to cultural competence training for clinicians and general protocol modifications (e.g., translation of materials, use of bilingual clinicians), this study's adaptations emphasize building partnerships with community constituents (e.g., health care organizations, pediatricians) to identify specific community needs and barriers. Through this inclusion of community recommendations, EP was able to more appropriately adapt its services to Latino family needs, increasing access to care for Latino families in poverty. Overall, the study findings provide compelling evidence for the incorporation of cultural modifications into evidence-based treatment to address the dearth of culturally adapted services for Latinos.

\section{Limitations}

One limitation of this study was the attrition rate $(32.1 \%)$ that occurred, which was similar to other PCT programs. Of important note, treatment completers did not differ from noncompleters on any of the study's outcome measures at pre-test suggesting that attrition may best be attributed to contextual factors as suggested by Nicholson et al. (1999). Second, despite the 
use of a waitlist-control group in which the delayed group waited four-six weeks, the immediate group did not always complete treatment within that six-week period, due to treatment-specific needs (e.g., child trauma history, medical issues). Therefore, additional time effects could not be isolated. Similarly, variable length of time between post-test and follow-up was a third limitation of this study. Consistent communication with families is a common challenge of providing treatment for low-income families. Therefore, while a four-six week follow-up was planned, typical duration between post-test and follow-up varied. A fourth limitation was the use of a convenience sample of self-referred participants, which may limit generalization. A fifth limitation was the moderate Kappa coefficients for the PCRS and GAF, though given the nature of those measures, moderate Kappa levels may be difficult to exceed.

Related to cultural modifications, the use of translators limited in-session communication between clinicians and families, often slowing the intervention process. Attempts to hire additional bilingual clinicians were unsuccessful, highlighting a growing need in the mental health field that must be addressed before the gap between the need and available treatment widens even further. In addition, this study's instruments have been normed with a relatively small portion of Latino families. Finally, the lack of bilingual supervisors may have impacted the effectiveness of supervision given potential clinician-supervisor communication issues.

\section{Conclusion/Implications for Future Research}

The successful outcomes of this study add two primary findings to the current literature: (a) evidence for the efficacy of a culturally-adapted model of the EP program for young Latino children and (b) support for the contributory effect of the EP in-home format as an effective treatment for child behavior problems. In light of these positive results, future research should address this study's limitations, with a particular emphasis on clinician effects, perhaps 
examining interpreter and bilingual clinician outcomes to clarify their impact on treatment. It also will behoove future research to compare Latino ethnicities (e.g., Mexican, Puerto Rican) to identify any within-group differences considering within-group heterogeneity of Latino populations as well as examine the impact of acculturation status on treatment. Perhaps assessing for outcome predictors also will provide insight into both the Early Pathways intervention and the cultural adaptation process. Finally, future research should assess what specific cultural modifications are beneficial through qualitative discussions with participants.

Future research also should examine two other areas of need. First, 30\% of participants reported a history of trauma, which required additional trauma-focused strategies. Second, poverty conditions and related contextual factors often served as barriers to treatment (e.g., medical needs, school concerns, financial limitations). While clinicians effectively managed these challenges by reviewing trauma-focused treatment and conducting advocacy efforts, it would be beneficial to incorporate and assess an evidence-based trauma-focused model into EP, as well as examine the impact of clinician advocacy on the treatment process and outcomes.

Finally, this study's findings suggest that the in-home format is an effective way to directly observe children in their natural environment, and help improve both child behavior and parent-child interactions. Given that the ITT analysis used in this study was more conservative than a dose-effect comparison, subsequently resulting in a higher attrition rate, future research should develop a more comprehensive definition of treatment adherence to better define treatment completion. One recommendation comes from Swift, Callahan, and Levine (2009), a multi-dimensional definition of treatment completion, incorporating treatment attendance, a reliable change index of a primary outcome measure, and a clinician observation of caregiver 
Early Pathways for Young Latino Children in Poverty 24

participation in treatment. Such a definition would assess participant outcomes on several facets of treatment, and the EP program is presently pursuing this approach for future studies. 


\section{References}

Ackerman, B. P., Brown, E., \& Izard, C. E. (2003). Continuity and change in levels of externalizing behavior in school of children from economically disadvantaged families. Child Development, 74, 694-709.

Alegría, M., Chatterji, P., Wells, K., Cao, Z., Chen, C., Takeuchi, D., ...Meng, X. (2008).

Disparity in depression treatment among racial and ethnic minority populations in the United States. Psychiatric Services, 59(11), 1264-1272.

American Psychiatric Association. (2000). Diagnostic and statistical manual of mental disorders ( $4^{\text {th }}$ ed., text rev.). Washington DC: Author.

Arcia, E., \& Johnson, A. (1998). When respect means to obey: Immigrant Mexican mothers' values for their children. Journal of Child and Family Studies, 7(1), 79-95.

Arciniega, G. M., Anderson, T. C., Tovar-Blank, Z. G., \& Tracey, T. J. G. (2008). Toward a fuller conception of machismo: Development of a traditional machismo and caballerismo scale. Journal of Counseling Psychology, 55(1), 19-33.

Author citation. (1994). [Title omitted for blind review].

Author citation. (1999). [Title omitted for blind review].

Author citation. (2003). [Title omitted for blind review].

Author citation. (2007). [Title omitted for blind review].

Author citation. (2009). [Title omitted for blind review].Author citation. (2009). [Title omitted for blind review].

Author citation. (2012). [Title omitted for blind review].

Author citation. (2013). [Title omitted for blind review].

Author citation. (2014). [Title omitted for blind review]. 
Author citation. (2014). [Title omitted for blind review]. Unpublished Training Manual.

Author citation. (in press). [Title omitted for blind review].

Barker, C. H., Cook, K. L., \& Borrego, Jr., J. (2010). Addressing cultural variables in parent training programs with Latino families. Cognitive and Behavioral Practice, 17, 157-166.

Barth, R. P., Greeson, J. K. P., Guo, S., Green, R. L., Hurley, S., \& Sisson, J. (2007). Outcomes for youth receiving intensive in-home therapy or residential care: A comparison using propensity scores. American Journal of Orthopsychiatry, 77(4), 497-505. doi: http://dx.doi.org/10.1037/0002-9432.77.4.497

Bernal, G., Bonilla, J., \& Bellido, C. (1995). Ecological validity and cultural sensitivity for outcome research: Issues for the cultural adaptation and development of psychosocial treatments with Hispanics. Journal of Abnormal Child Psychology, 23, 67-82.

Breitenstein, S. M., Gross, D., Ordaz, I., Julion, W., Garvey, C., \& Ridge, A. (2007). Promoting mental health in early childhood programs serving families from low-income neighborhoods. Journal of the American Psychiatric Nurses Association, 13(5), 313-320.

Brotman, L. M., Calzada, E., Huang, K., Kingston, S., Dawson-McClure, S., Kamboukos, D., ...Petkova, E. (2011). Promoting effective parenting practices and preventing child behavior problems in school among ethnically diverse families from underserved, urban communities. Child Development, 82(1), 258-276.

Calderón-Tena, C. O., Knight, G. P., \& Carlo, G. (2011). The socialization of prosocial behavioral tendencies among Mexican American adolescents: The role of familism values. Cultural Diversity and Ethnic Minority Psychology, 17(1) 98-106. 
Castillo, L. G., \& Cano, M. A. (2007). Mexican American psychology: Theory and clinical application. In C. Negy, (Ed.), Cross-cultural psychotherapy: Toward a critical understanding of diverse clients (pp. 85-102). Reno, NV: Bent Tree Press.

Crawley, S. B., \& Spiker, D. (1983). Mother-child interactions involving two-year-olds with Down syndrome: A look at individual differences. Child Development, 54, 1312-1323.

Domenech-Rodríguez, M., \& Wieling, E. (2004). Developing culturally appropriate, evidencebased treatments for interventions with ethnic minority populations. In M. Rastogin \& E. Wieling (Eds.), Voices of color: First person accounts of ethnic minority therapist (pp. 313-333). Thousand Oaks, CA: Sage.

Dumas, J. E., Arriaga, X. B., Moreland-Begle, A. M., \& Longoria, Z. N. (2011). Child and parental outcomes of a group parenting intervention for Latino families: A pilot study of the CANNE program. Cultural Diversity and Ethnic Minority Psychology, 17(1), 107115.

Eyberg, S. M., \& Boggs, S. R. (1989). Parent training for oppositional-defiant preschoolers. In C. E. Schaefer \& J. M. Briesmeister (Eds.), Handbook of parent training: Parents as cotherapists for children's behavior problems (pp. 105-132). New York: Wiley.

Eyberg, S. M., Funderburk, B. W., Hembree-Kigin, T. L., McNeil, C. B., Querido, J. G., \& Hood, K. K. (2001). Parent-Child Interaction Therapy with behavior problem children: One and two year maintenance of treatment effects in the family. Child \& Family Behavior Therapy, 23(4), 1-20.

Eyberg, S. M., \& Pincus, D. (1999). Eyberg Child Behavior Inventory and Sutter-Eyberg Student Behavior Inventory-Revised: Professional manual. Lutz, Florida: Psychological Assessment Resources, Inc. 
Early Pathways for Young Latino Children in Poverty 28

Fernandez, M. A., Butler, A. M., \& Eyberg, S. M. (2011). Treatment outcome for low socioeconomic status African American families in Parent-Child Interaction Therapy: A pilot study. Child \& Family Behavior Therapy, 33, 32-48.

Foucault, D. C., \& Schneider, B. H. (2009). Parenting values and parenting stress among impoverished village and middle-class small city mothers in the Dominican Republic. International Journal of Behavioral Development, 33(5), 440-450.

Griner, D., \& Smith, T. B. (2006). Culturally adapted mental health interventions: A metaanalytic review. Psychotherapy: Theory, Research, Practice, Training, 43(4), 531-548.

Gross, D., Garvey, C., Julion, W., Fogg, L., Tucker, S., \& Mokros, H. (2009). Efficacy of the Chicago Parent Program with low-income African-American and Latino parents of young children. Prevention Science, 10, 54-65.

Grossman, D. (1994). Enhancing your 'cultural competence.' American Journal of Nursing, 94(7), 58-62.

Lakes, K. D., Kettler, R. J., Schmidt, J., Haynes, M., Feeney-Kettler, K., Kamptner, L., ...Tamm, L. (2009). The CUIDAR early intervention parent training program for preschoolers at risk for behavioral disorders: An innovative practice for reducing disparities in access to service. Journal of Early Intervention, 31(2), 167-178.

Marín, G., Sabogal, F., Marín, B. V., Otero-Sabogal, R., \& Perez-Stable, E. J. (1987). Development of a short acculturation scale for Hispanics. Hispanic Journal of Behavioral Sciences, 9, 183-205.

Matos, M., Bauermeister, J. J., \& Bernal, G. (2009). Parent-Child Interaction Therapy for Puerto Rican preschool children with ADHD and behavior problems: A pilot efficacy study. Family Process, 48(2), 232-252. 
Early Pathways for Young Latino Children in Poverty 29

McCabe, K., \& Yeh, M. (2009). Parent-Child Interaction Therapy for Mexican Americans: A randomized clinical trial. Journal of Clinical Child \& Adolescent Psychology, 38(5), 753759.

Peña, J. B., Kuhlberg, J. A., Zayas, L. H., Baumann, A. A., Gulbas, L., Hausmann-Stabile, C., \& Nolle, A. P. (2011). Familism, family environment, and suicide attempts among Latina youth. Suicide and Life-Threatening Behavior, 41(3), 330-341.

Qi, C. H., \& Kaiser, A. P. (2003). Behavior problems of preschool children from low-income families: Review of the literature. Topics in Early Childhood Special Education, 23(4), $188-216$.

Roberts, C., Mazzucchelli, T., Studman, L., \& Sanders, M. R. (2006). Behavioral family intervention for children with developmental disabilities and behavioral problems. Journal of Clinical and Adolescent Psychology, 35, 180-193.

Sanders, M. R. (1999). Triple P-Positive parenting program: Towards an empirically validated multilevel parenting and family support strategy for the prevention of behavior and emotional problems in children. Clinical Child and Family Psychology Review, 2, 71-90.

Smith, T. B., Domenech-Rodríguez, M., \& Bernal, G. (2011). Culture. Journal of Clinical Psychology, 67(2), 166-175.

Spencer, M. S., Kohn, L. P., \& Woods, J. R. (2002). Labeling vs. early identification: The dilemma of mental health service utilization among low-income African American children. African American Research Perspectives, 8, 1-14.

Swift, J. K., Callahan, J., \& Levine, J. C. (2009). Using clinically significant change to identify premature termination. Psychotherapy: Theory, Research, Practice, Training, 46(3), 328335. doi: 10.1037/a0017003 
Unger, J. B., Ritt-Olson, A., Teran, L., Huang, T., Hoffman, B., \& Palmer, P. (2002). Cultural values and substance use in a multiethnic sample of California adolescents. Addictions Research Theory, 10, 257-280.

U.S. Census Bureau. (2001). Poverty in the United States: 2000. Washington DC: U.S. Government Printing Office. Retrieved on November 9, 2011, from http://www.census.gov/prod/2001pubs/p60-214.pdf

U.S. Census Bureau. (2011). Income, poverty, and health insurance coverage in the United States: 2010. Washington DC: U.S. Government Printing Office. Retrieved on November 9, 2011, from http://www.census.gov/prod/2011pubs/p60-239.pdf

Viera, A. J., \& Garrett, J. M. (2005). Understanding interobserver agreement: The Kappa statistic. Research Series, 37(5), 360-363.

Webster-Stratton, C. (1992). The Incredible Years: A trouble shooting guide for parents of children age 3-8 years. Toronto: Umbrella Press.

Webster-Stratton, C., Reid, M. J., \& Hammond, M. (2004). Treating children with early-onset conduct problems: Intervention outcomes for parent, child, and teacher training. Journal of Clinical Child and Adolescent Psychology, 33(1), 105-124.

Webster-Stratton, C., Reid, M. J., \& Stoolmiller, M. (2008). Preventing conduct problems and improving school readiness: Evaluation of the Incredible Years Teacher and Child Training Programs in high-risk schools. The Journal of Child Psychology and Psychiatry, $49(5), 471-488$

Wood, S., Barton, K., \& Schroeder, C. (1988). In-home treatment of abusive families: Cost and placement at one year. Psychotherapy: Theory, Research, Practice, Training, 25(3), 409414. doi: http://dx.doi.org/10.1037/h0085362. 


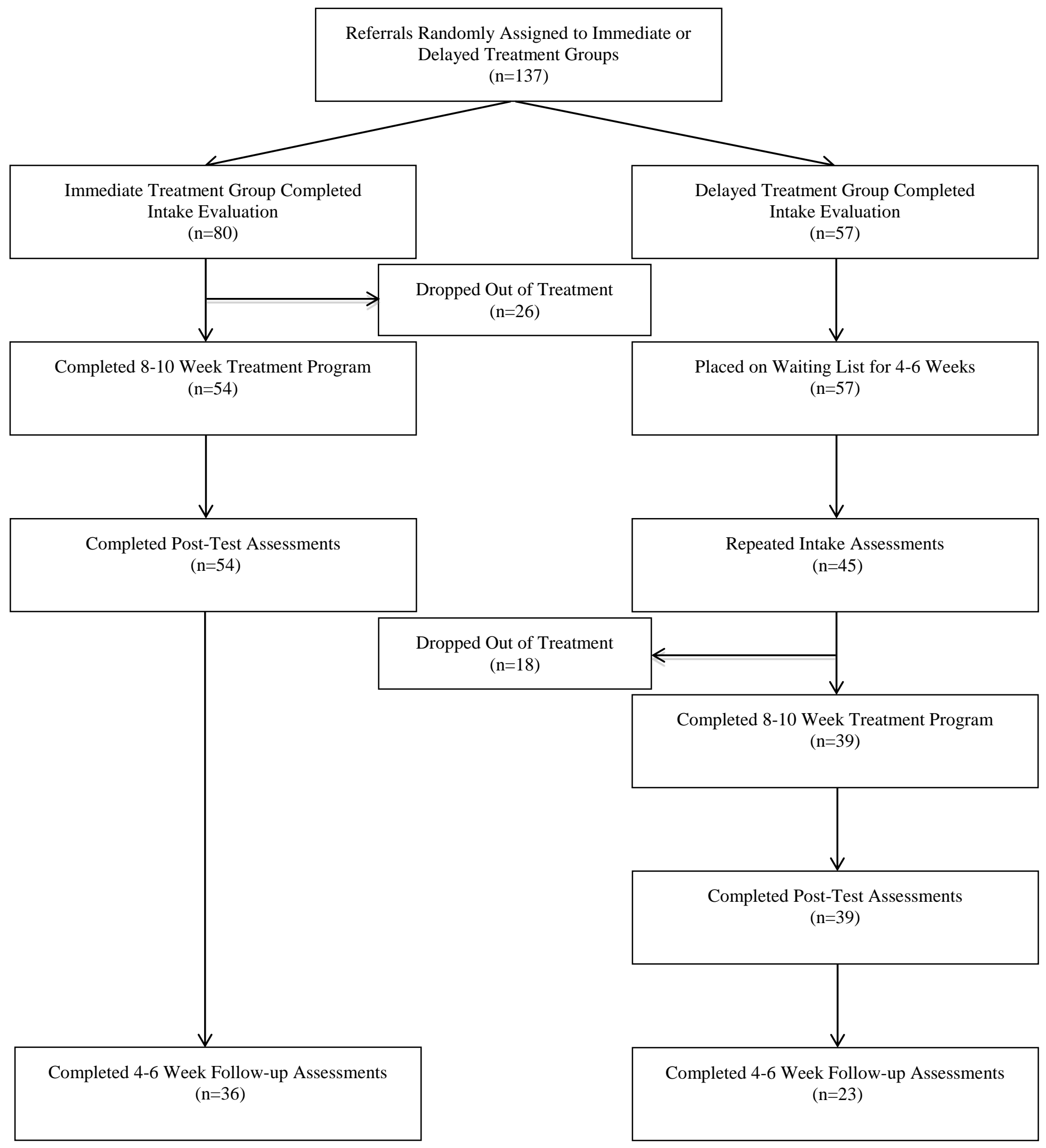

Figure 1

Participant Flowchart from Random Group Assignment through Short-Term Follow-Up Evaluations 
Table 1

MANCOVAs with Pairwise Comparisons for Immediate vs. Delayed Groups at Pre-test, Post-test/Pre-test 2, and Follow-up

\begin{tabular}{|c|c|c|c|c|c|c|c|c|c|c|c|}
\hline & $\begin{array}{c}\text { Immediate } \\
\text { Pre-test }\end{array}$ & & $\begin{array}{l}\text { Delayed } \\
\text { Pre-test }\end{array}$ & & $\begin{array}{l}\text { Immediate } \\
\text { Post-test }\end{array}$ & & $\begin{array}{l}\text { Delayed } \\
\text { Pre-test } 2\end{array}$ & & & $\begin{array}{l}\text { Delayed } \\
\text { Post-test }\end{array}$ & \\
\hline Measures & $M$ & $S D$ & $M$ & $S D$ & $M$ & $S D$ & $M$ & $S D$ & $d$ & $M$ & $S D$ \\
\hline \multicolumn{12}{|l|}{ ECBS } \\
\hline Pro-Social & 21.61 & 3.34 & 22.32 & 2.69 & $25.15^{*}$ & 2.92 & 23.18 & 2.80 & .96 & 25.17 & 2.23 \\
\hline Challenging & 22.76 & 4.48 & 22.72 & 4.14 & $16.74 *$ & 4.25 & 21.96 & 4.12 & 1.88 & 17.03 & 3.85 \\
\hline \multicolumn{12}{|l|}{ PCPA } \\
\hline Child & 7.27 & 2.27 & 6.76 & 2.44 & $9.13 *$ & 1.07 & 6.93 & 2.44 & 1.07 & 8.19 & 2.42 \\
\hline Parent & 8.26 & 2.10 & 7.50 & 2.31 & $10.84 *$ & 1.26 & 7.63 & 2.37 & 1.65 & 10.12 & 2.01 \\
\hline \multicolumn{12}{|l|}{$\mathrm{PBC}$} \\
\hline Discipline & 14.93 & 4.78 & 14.93 & 4.58 & $12.76^{*}$ & 3.34 & 14.57 & 4.62 & .84 & 12.37 & 3.38 \\
\hline Nurturing & 28.80 & 5.53 & 28.98 & 5.56 & $30.57 *$ & 5.77 & 28.18 & 6.14 & .60 & 29.60 & 5.97 \\
\hline GAF & 56.44 & 7.64 & 56.81 & 8.68 & $74.83 *$ & 8.75 & 57.24 & 7.36 & 2.71 & 74.73 & 9.59 \\
\hline PCRS & 54.57 & 12.28 & 56.84 & 11.94 & $77.74 *$ & 11.00 & 57.68 & 9.69 & 2.61 & 76.17 & 8.78 \\
\hline
\end{tabular}

Note: $*_{p}<.001$. The notation $d^{l}$ refers to effect size of MANCOVA comparisons at post-test with pre-test scores as covariates. 